Infinite Dimensional Analysis, Quantum Probability and Related Topics

(C) World Scientific Publishing Company

\title{
QUANTUM MULTIPOLE NOISE AND GENERALIZED QUANTUM STOCHASTIC EQUATIONS
}

\author{
A. N. PECHEN* and I. V. VOLOVICH ${ }^{\dagger}$ \\ Steklov Mathematical Institute, Russian Academy of Sciences, \\ Gubkin St. 8, GSP-1, 117966, Moscow, Russia
}

\begin{abstract}
A notion of quantum multipole (in particular, dipole) noise is considered. Quantum dipole noise is an analogue of quantum white noise but it acts in a Fock space with indefinite metric. Quantum white noise describes the leading term in the stochastic limit approximation to quantum dynamics while quantum multipole noise describes corrections to the leading term. We obtain and study generalized quantum stochastic equations describing corrections to the stochastic limit which include quantum dipole noise.

Keywords: multipole noise, white noise, dipole noise, quantum stochastic equations
\end{abstract}

\section{Introduction}

There are numerous works devoted to the studying of the long time behavior in quantum theory. Recently an exact formula for the time dependence of certain matrix elements (ABC-formula) has been obtained in 1].

The leading term in the weak coupling - large time regime in this formula corresponds to the stochastic limit (see [2]). In the operator formalism the stochastic limit is described by the quantum white noise and corresponding quantum stochastic differential equations [3]- 6 .

To describe corrections to the stochastic limit one needs a new operator structure. For free fields an appropriate new notion was introduced in [7. We call it the quantum dipole noise. In this paper we first study mathematical properties of the quantum dipole noise and then consider interacting case and show that the quantum dipole noise describes first order corrections to the stochastic limit. We introduce quantum multipole noise which describes corrections of any order to the stochastic limit. Moreover we introduce a new type of quantum stochastic differential equations which involves quantum dipole noise. These quantum stochastic differential equations we call generalized quantum stochastic differential equations.

${ }^{*}$ E-mail: pechen@mi.ras.ru
${ }^{\dagger}$ E-mail: volovich@mi.ras.ru 
Let us remind that the quantum white noise is an operator valued distribution with commutation relations proportional to Dirac's $\delta$-function. The simplest example of the quantum white noise is given by the following commutation relations

$$
\left[b(t), b^{+}(\tau)\right]=\delta(\tau-t) .
$$

The white noise operators are the subject of investigation in numerous works [2]- $[6]$.

In the present article we study higher order corrections to the stochastic limit. We show that a new mathematical object: dipole noise operators - naturally arises as the second order terms in the expansion of collective operators $a^{ \pm}\left(k, t / \lambda^{2}\right)$ in the series with respect to the $\lambda$. Here $a^{ \pm}(k, t):=e^{ \pm i \omega(k) t} a^{ \pm}(k)$, where $a^{-}(k)$ and $a^{+}(k)$ are bosonic annihilation and creation operators.

An operator valued distribution with commutation relations proportional to derivative of Dirac's $\delta$-function has being introduced in [7. We call it the quantum dipole noise. The simplest example of quantum dipole noise is defined by the commutation relations

$$
\left[c(t), c^{+}(\tau)\right]=i \delta^{\prime}(\tau-t) .
$$

We introduce and study also further generalizations of the white and dipole noise operators which we call multipole noise operators. They satisfy the commutation relations

$$
\left[c_{m}(t), c_{n}^{+}(\tau)\right]=\delta_{n, m} i^{n} \delta^{(n)}(\tau-t),
$$

where $m, n=0,1, \ldots, c_{0}(t) \equiv b(t)$ and $c_{1}(t) \equiv c(t)$.

White noise operators arise as the first order terms in the expansion of the free fields in the series with respect to $\lambda$ and given by the following limit (in the sense of convergence of correlators, see [2])

$$
\lim _{\lambda \rightarrow 0} \frac{1}{\lambda} a^{ \pm}\left(k, t / \lambda^{2}\right)=b^{ \pm}(k, t) .
$$

Here $b^{ \pm}(k, t)$ are the white noise operators. They satisfy the following commutation relations

$$
\left[b^{-}(k, t), b^{+}(p, \tau)\right]=2 \pi \delta(\omega(k)) \delta(\tau-t) \delta(p-k) .
$$

Next terms of the expansion of the collective operators in the series with respect to the $\lambda$ have being found in [7. They are the dipole noise operators $c^{ \pm}(k, t)$ satisfying the following commutation relations

$$
\left[c^{-}(k, t), c^{+}(p, \tau)\right]=2 \pi i \delta^{\prime}(\omega(k)) \delta^{\prime}(\tau-t) \delta(p-k) .
$$

We will also use notations $a(k) \equiv a^{-}(k), b(k, t) \equiv b^{-}(k, t)$ and $c(k, t) \equiv c^{-}(k, t)$.

In section 2 we will construct a representation of dipole noise (1.2) in a pseudoHilbert space with indefinite metric. We will show that the quantum dipole noise is a well defined operator valued distribution in a Fock space with indefinite metric. Notice that spaces with indefinite metric are widely used in quantum field theory for quantization of electromagnetic and other gauge fields (Gupta-Bleuler formalism, [8], see also [9] 10]). 
Using multipole (white and dipole) noise operators one can make the formal expansion

$$
a^{ \pm}\left(k, t / \lambda^{2}\right)=\lambda b^{ \pm}(k, t)+\lambda^{2} c^{ \pm}(k, t)+o\left(\lambda^{2}\right) .
$$

The base for this expansion is the following expansion (in the sense of distributions)

$$
\frac{1}{\lambda^{2}} e^{i t x / \lambda^{2}}=2 \pi \delta(x) \delta(t)+\lambda^{2} 2 \pi i \delta^{\prime}(x) \delta^{\prime}(t)+o\left(\lambda^{2}\right),
$$

where $x$ and $t$ are real variables.

Using expansion (1.7) of the collective (in this case free) operators we obtain the equation for the higher order corrections to the stochastic limit of the evolution operator $U_{\lambda}(t)$ of system interacting with Bose field. Then we bring this equation to the normally ordered form which is convenient for calculation of corrections for matrix elements of the evolution operator.

One has the expansion for the rescaled evolution operator into the series at the coupling constant $\lambda$

$$
U_{\lambda}\left(t / \lambda^{2}\right)=U_{0}(t)+\lambda U_{1}(t)+\ldots
$$

The normal form of the equation for the evolution operator $U_{0}(t)$ in the stochastic limit is equivalent to the quantum stochastic differential equation in the sense of [1] and looks like

$$
d U_{0}(t)=\left(D d B_{t}^{+}-D^{+} d B_{t}-\gamma D^{+} D d t\right) U_{0}(t) .
$$

Here we use standard notations $d B_{t}$ and $d B_{t}^{+}$for stochastic differentials of quantum Brownian motion; $D$ and $D^{+}$are bounded operators in system Hilbert space and $\gamma$ is a complex number.

We will obtain quantum differential equations of a new form which include quantum multipole noise. They will describe the higher order corrections to the stochastic limit. For example for the first order correction $U_{1}(t)$ to the evolution operator one has the generalized quantum stochastic differential equation

$$
d U_{1}(t)=\left(D d C_{t}^{+}-D^{+} d C_{t}\right) U_{0}(t)+\left(D d B_{t}^{+}-D^{+} d B_{t}-\gamma D^{+} D d t\right) U_{1}(t) .
$$

We call it generalized quantum stochastic differential equation because it contains stochastic differentials of dipole noise which we denoted as $d C_{t}$ and $d C_{t}^{+}$. These operators are defined in a Fock space with indefinite metric.

In Sect. 2 quantum dipole noise operators in the Fock space with indefinite metric are defined. An expansion of the free fields into the series containing multipole noise operators is obtained in Sect. 3. Dipole noise on the simplex is considered in Sect. 4 wcorrections to the Green function are described in Sect. 5. Finally generalized quantum stochastic differential equations describing corrections to the stochastic limit are presented in Sect. 6-9.

\section{Quantum Dipole Noise in Fock Space with Indefinite Metric}

The commutation relations (1.2) for the dipole noise mean that one has a family of operators $c_{g}$ parameterized by complex valued functions of real argument $g(t)$ from 
some space of functions (for example from Schwartz space of functions $S(\mathbb{R})$ ) with commutation relations

$$
\left[c_{f}, c_{g}^{+}\right]=<f, g>
$$

where

$$
<f, g>=i \int \overline{f^{\prime}(t)} g(t) d t .
$$

Notice that the inner product $\langle\cdot, \cdot\rangle$ is non-positively defined, i.e. it is an indefinite inner product. In fact, for $f(t)=u(t)+i v(t)$ with $u(t)$ and $v(t)$ are real valued functions one has

$$
<f, f>=-2 \int u^{\prime}(t) v(t) d t .
$$

This quantity can be positive or negative. Therefore the natural space of representation of algebra (2.10) is a vector space with indefinite inner product.

Let us describe a general construction of a Fock space with indefinite metric (see [9, 10]). Let $\mathcal{H}_{1}$ be a Hilbert space over field $\mathbb{C}$ and $(\cdot, \cdot)_{\mathcal{H}_{1}}$ is the inner product in $\mathcal{H}_{1}$. This inner product induces in $\mathcal{H}_{1}$ a structure of complex Frećhet space with Hilbert topology. An indefinite inner product on $\mathcal{H}_{1}$ is a hermitian sesquilinear form, i.e. a map $\mathcal{H}_{1} \times \mathcal{H}_{1} \rightarrow \mathbb{C}$ with the properties

$$
\begin{gathered}
<f, \alpha g+\beta h>=\alpha<f, g>+\beta<f, h> \\
<f, g>=\overline{<g, f>}
\end{gathered}
$$

for all $f, g, h \in \mathcal{H}_{1}$ and $\alpha, \beta \in \mathbb{C}$. The form $\langle\cdot, \cdot\rangle$ is non necessarily positively defined. It is called an indefinite metric. We suppose that if $F(\cdot)$ is a continuous linear functional over the topological space $\mathcal{H}_{1}$ then it can be uniquely represented in the form

$$
F(g)=<f, g>, \quad g \in \mathcal{H}_{1}
$$

for some $f \in \mathcal{H}_{1}$. Moreover, we suppose that for any $f \in \mathcal{H}_{1}$ Eq. (2.12) determines a linear continuous functional $F(g)$ over $\mathcal{H}_{1}$. If (2.12) holds then we say that the Hilbert topology is consistent with the indefinite inner product. In this case the complex Frećhet space with Hilbert topology and with the indefinite metric is being called a pseudo-Hilbert space. The condition of 2.12) is equivalent to existence of a bounded hermitian with respect to the inner product $(\cdot, \cdot)_{\mathcal{H}_{1}}$ linear operator $\eta: \mathcal{H}_{1} \rightarrow \mathcal{H}_{1}$ such that the inverse operator $\eta^{-1}$ exists, bounded and moreover $\eta$ satisfies the following property:

$$
<f, g>=(f, \eta g)_{\mathcal{H}_{1}}, \quad \forall f, g \in \mathcal{H}_{1} .
$$

Notice that one can vary the inner product in the definition of Hilbert topology. In fact, two different inner products in $\mathcal{H}_{1}$, say $(\cdot, \cdot)_{\mathcal{H}_{1}}$ and $(\cdot, \cdot)_{\mathcal{H}_{1}}^{\prime}$, induce the same topology if $\exists C, C^{\prime}>0$ :

$$
C(f, f)_{\mathcal{H}_{1}} \leq(f, f)_{\mathcal{H}_{1}}^{\prime} \leq C^{\prime}(f, f)_{\mathcal{H}_{1}} .
$$

Moreover, one can choose the inner product in such a way that the operator $\eta$ satisfies an additional condition

$$
\eta^{2}=1
$$


We will denote in this paper as $(\cdot, \cdot)_{\mathcal{H}_{1}}$ the inner product for which operator $\eta$ satisfies (2.13).

Let $\mathcal{H}_{1}^{\otimes_{s}^{n}}:=\operatorname{Sym} \mathcal{H}_{1} \otimes \ldots \otimes \mathcal{H}_{1}$ be a symmetric tensor product of $n$ copies of $\mathcal{H}_{1}$ for $n \geq 1$ and $\mathbb{C}$ for $n=0$. Operator of symmetrization acts on $\tilde{f}_{n}=f^{(1)} \otimes \ldots \otimes f^{(n)}$ as

$$
\text { Sym } \tilde{f}_{n}=\frac{1}{n !} \sum_{\pi} f^{(\pi(1))} \otimes \ldots \otimes f^{(\pi(n))}=: f_{n}
$$

with summation over all permutations of the set $\{1, \ldots, n\}$. The indefinite inner product of two vectors $f_{n}, g_{n} \in \mathcal{H}_{1}^{\otimes_{s}^{n}}$ for $n>0$ is given by

$$
<f_{n}, g_{n}>:=\left(f_{n}, \eta^{\otimes^{n}} g_{n}\right)_{\mathcal{H}_{1}^{\otimes_{s}^{n}}}
$$

where $\eta^{\otimes^{n}}:=\eta \otimes \ldots \otimes \eta$ is the tensor product of $n$ copies of $\eta$. For $n=0$ we define

$$
<f_{0}, g_{0}>:=\bar{f}_{0} g_{0}
$$

Then we can define the Boson Fock space with indefinite metric

$$
\mathcal{F}\left(\mathcal{H}_{1}\right)=\bigoplus_{n=0}^{\infty} \mathcal{H}_{1}^{\otimes_{s}^{n}}
$$

We mean that any vector $\phi \in \mathcal{F}\left(\mathcal{H}_{1}\right)$ is a sequence of the form

$$
\phi=\left(f_{0}, f_{1}, \ldots, f_{n}, \ldots\right)
$$

with the convergent series

$$
\|\phi\|^{2}=\sum_{n=0}^{\infty}\left(f_{n}, f_{n}\right)_{\mathcal{H}_{1}^{\otimes_{s}^{n}}}<\infty
$$

Here $f_{0} \in \mathbb{C}$ is a complex number, $f_{n}$ given by (2.14) and is being called the $n^{-}$ particle component of the vector $\phi$. The indefinite inner product of two vectors $\phi=\left(f_{0}, f_{1}, \ldots\right)$ and $\psi=\left(g_{0}, g_{1}, \ldots\right)$ is given by

$$
<\phi, \psi>=\sum_{n=0}^{\infty}<f_{n}, g_{n}>.
$$

The series is convergent because $<f_{n}, g_{n}>=\left(f_{n}, \eta^{\otimes^{n}} g_{n}\right)_{\mathcal{H}_{1}}$ and $\eta^{2}=1$.

Creation and annihilation operators $c_{f}^{+}$and $c_{f}$ act on $n$-particle component of the vector $\phi$ by usual formulae

$$
\begin{gathered}
\left(c_{f}^{+} f_{n}\right)_{n+1}=\sqrt{n+1} \operatorname{Sym} f \otimes f_{n} \\
\left(c_{f} f_{n}\right)_{n-1}=\frac{1}{\sqrt{n}} \sum_{i=1}^{n}<f, f^{(i)}>\operatorname{Sym} f^{(1)} \otimes \ldots \hat{f}^{(i)} \ldots \otimes f^{(n)} .
\end{gathered}
$$


Here $\hat{f}^{(i)}$ means that $f^{(i)}$ is missed. One has the canonical commutation relations $(\mathrm{CCR})$

$$
\left[c_{f}, c_{g}^{+}\right]=<f, g>
$$

and also on finite vectors one has the relation

$$
<c_{f} \phi, \psi>=<\phi, c_{f}^{+} \psi>
$$

which means that the annihilator $c_{f}$ is the adjoint of the creator $c_{f}^{+}$with respect to the inner product $\langle\cdot, \cdot\rangle$.

In order to construct a representation of algebra (2.10) let us choose $\mathcal{H}_{1}$ to be the completion of the Schwartz space $S(\mathbb{R})$ with respect to inner product

$$
(f, g)_{\mathcal{H}_{1}}:=\frac{1}{2 \pi} \int|\tau| \overline{\tilde{f}(\tau)} \tilde{g}(\tau) d \tau
$$

Here

$$
\tilde{f}(\tau)=\int e^{i x \tau} f(x) d x
$$

is the Fourier transform of $f(x)$. We introduce an indefinite inner product on $\mathcal{H}_{1}$ by the formula

$$
<f, g>=i \int \overline{f^{\prime}(t)} g(t) d t
$$

which can be rewritten in the form

$$
<f, g>=\frac{1}{2 \pi} \int \tau \overline{\tilde{f}(\tau)} \tilde{g}(\tau) d \tau .
$$

In this case the operator $\eta$ acts on the Fourier transform of the function $f(x)$ as

$$
(\eta \tilde{f})(\tau)=\operatorname{sign}(\tau) \tilde{f}(\tau)
$$

where $\operatorname{sign}(\tau)=1$ if $\tau \geq 0$ and -1 in the opposite case. Clearly this operator satisfies (2.13) and all conditions which are necessary to guarantee 2.12). Therefore the Hilbert topology which given by (2.15) is consistent with the indefinite metric (2.16).

Any vector $\phi \in \mathcal{F}\left(\mathcal{H}_{1}\right)$ is a sequence of (symmetric) functions,

$$
\phi=\left(f_{0}, f_{1}\left(t_{1}\right), \ldots, f_{n}\left(t_{1}, \ldots, t_{n}\right), \ldots\right) .
$$

Here $f_{0} \in \mathbb{C}$ is a complex number as above.

According to general construction described above creation and annihilation dipole noise operators $c_{f}^{+}$and $c_{f}$ act on $n$-particle component of the vector $\phi$ by formulae

$$
\begin{gathered}
\left(c_{f}^{+} f_{n}\right)_{n+1}\left(t_{1}, \ldots, t_{n+1}\right)=\frac{1}{\sqrt{n+1}} \sum_{i=1}^{n+1} f\left(t_{i}\right) f_{n}\left(t_{1}, \ldots, \hat{t}_{i}, \ldots, t_{n+1}\right) . \\
\left(c_{f} f_{n}\right)_{n-1}\left(t_{1}, \ldots, t_{n-1}\right)=i \sqrt{n} \int \overline{f^{\prime}(t)} f_{n}\left(t, t_{1}, \ldots, t_{n-1}\right) d t
\end{gathered}
$$

Here $\hat{t}_{i}$ means that argument $t_{1}$ is missed. 
Remark 2.1. Motivated by the investigation of the space with the sesquilinear form (2.11) let us note that it is interesting to consider the following approach to solution of the Dirichlet problem. Let us consider the Laplace equation

$$
-\Delta u(x)=f(x), \quad x \in \Omega \subset \mathbb{R}^{d}
$$

where $\Omega$ is an arbitrary domain in $\mathbb{R}^{d}$. Denote $\mathcal{H}$ the Hilbert space obtained after the completion and taking the factor space of the space of test functions with compact support $\mathcal{D}(\Omega)$ with the scalar product

$$
(\varphi, \psi)_{\mathcal{H}}=\int_{\Omega} \nabla \bar{\varphi} \nabla \psi d x .
$$

We write Eq. (2.17) in the form

$$
(u, \varphi)_{\mathcal{H}}=(f, \varphi), \quad \varphi \in \mathcal{D}(\Omega)
$$

where

$$
(f, \varphi)=\int_{\Omega} \overline{f(x)} \varphi(x) d x .
$$

Proposition 2.1. Let $f \in L_{l o c}(\Omega)$ and there exists a constant $C_{\Omega}>0$ such that

$$
|(f, \varphi)|^{2} \leq C_{\Omega}(\varphi, \varphi)_{\mathcal{H}} \quad \forall \varphi \in \mathcal{D}(\Omega) .
$$

Then there exists a unique solution $u \in \mathcal{H}$ of Eq.(2.18).

\section{Multipole Noise}

In this section we will show that the quantum multipole noise naturally arises from studying of the higher order corrections to the stochastic limit.

Let $L^{2}\left(\mathbb{R}^{d}\right)$ be the Hilbert space (with inner product $(\cdot, \cdot)$ ) of square integrable on $\mathbb{R}^{d}$ functions. Denote then

$$
\mathcal{F}=\bigoplus_{n=0}^{\infty} L^{2}\left(\mathbb{R}^{d}\right)^{\otimes_{s}^{n}}
$$

the Boson Fock space and $a(f), a^{+}(g)$ are annihilation and creation operators in $\mathcal{F}$ with commutation relations

$$
\left[a(f), a^{+}(g)\right]=(f, g) .
$$

Here $f, g \in L^{2}\left(\mathbb{R}^{d}\right)$ and we will use the notation

$$
a(f)=\int \bar{f}(k) a(k) d k, \quad k \in \mathbb{R}^{d} .
$$

For a given complex valued test function $g(k)$ on $\mathbb{R}^{d}$ and for smooth real valued function $\omega(k)$ define the (smeared) time dependent creation and annihilation operators as

$$
A^{+}(t)=\int g(k) e^{i \omega(k) t} a^{+}(k) d k, \quad A(t)=\int \bar{g}(k) e^{-i \omega(k) t} a(k) d k .
$$


The test function $g(k)$ in physical applications plays a role of formfactor describing structure of interaction. The function $\omega(k)$ has meaning of dispersion law so that these operators are free evolutions of smeared creation and annihilation operators. We assume that the surface $\omega(k)=0$ is nondegenerate, i.e. $\operatorname{grad} \omega \neq 0$ on the surface $\omega(k)=0$.

Let us consider the commutator of rescalled creation and annihilation operators

$$
A_{\lambda}(t):=\frac{1}{\lambda} A\left(t / \lambda^{2}\right), \quad A_{\lambda}^{+}(t):=\frac{1}{\lambda} A^{+}\left(t / \lambda^{2}\right)
$$

(we call them collective operators), where $\lambda$ is a real parameter. One has

$$
\left[A_{\lambda}(t), A_{\lambda}^{+}(\tau)\right]=\frac{1}{\lambda^{2}} \int d k|g(k)|^{2} e^{i \frac{\omega(k)(\tau-t)}{\lambda^{2}}} .
$$

In order to investigate the behavior of this commutator for small $\lambda$ let us consider the sequence of functions on $\mathbb{R}^{2}$ indexed by real number $\lambda \neq 0$

$$
f_{\lambda}(x, t)=\frac{1}{\lambda^{2}} e^{i x t / \lambda^{2}} .
$$

For any $\lambda \neq 0 f_{\lambda}(x, t)$ is locally integrable. Therefore it defines a distribution from $S^{\prime}\left(\mathbb{R}^{2}\right)$. In the sense of distributions one has the relation $([2])$

$$
\lim _{\lambda \rightarrow 0} f_{\lambda}(x, t)=2 \pi \delta(x) \delta(t) .
$$

We will prove the following generalization of this relation.

Theorem 3.1. One has the asymptotic expansion (in the sense of distributions)

$$
\frac{1}{\lambda^{2}} e^{i x t / \lambda^{2}}=2 \pi \sum_{n=0}^{\infty} \frac{\left(i \lambda^{2}\right)^{n}}{n !} \delta^{(n)}(x) \delta^{(n)}(t) .
$$

The asymptotic series 3.21) means that for any test functions $f$ and $\phi$ from the Schwartz space $S(\mathbb{R})$ and for any $N \in \mathbb{N}$

$$
\int \frac{1}{\lambda^{2}} e^{i x t / \lambda^{2}} f(x) \phi(t) d x d t-2 \pi \sum_{n=0}^{N} \frac{\left(i \lambda^{2}\right)^{n}}{n !} f^{(n)}(0) \phi^{(n)}(0)=o\left(\lambda^{2 N}\right) .
$$

Proof. Let $f(x)$ and $\phi(t)$ be test functions from the Schwartz space $S(\mathbb{R})$. Then one has

$$
\int \frac{1}{\lambda^{2}} e^{i x t / \lambda^{2}} f(x) \phi(t) d x d t=\int \tilde{f}(\tau) \phi\left(\lambda^{2} \tau\right) d \tau .
$$

Here

$$
\tilde{f}(\tau)=\int e^{i x \tau} f(x) d x
$$

is the Fourier transform of $f(x)$. It is well-known (see [12]) that $\tilde{f}(\tau) \in S(\mathbb{R})$ and the inverse Fourier transform is given by the formula

$$
f(x)=\frac{1}{2 \pi} \int e^{-i x \tau} \tilde{f}(\tau) d \tau .
$$


Since $\phi(t) \in S(\mathbb{R})$ one can write the Taylor expansion for $\phi\left(\lambda^{2} \tau\right)$ in the Lagrange form

$$
\phi\left(\lambda^{2} \tau\right)=\phi(0)+\sum_{n=1}^{N} \frac{\left(\lambda^{2} \tau\right)^{n}}{n !} \phi^{(n)}(0)+\frac{\left(\lambda^{2} \tau\right)^{N+1}}{(N+1) !} \phi^{(N+1)}\left(\xi_{\lambda}(\tau)\right)
$$

where $\xi_{\lambda}(\tau) \in\left(0, \lambda^{2} \tau\right)$. Using this expansion one gets

$$
\begin{gathered}
\int \tilde{f}(\tau) \phi\left(\lambda^{2} \tau\right) d \tau-\sum_{n=0}^{N} \frac{\lambda^{2 n}}{n !} \phi^{(n)}(0) \int \tau^{n} \tilde{f}(\tau) d \tau \\
=\frac{\lambda^{2(N+1)}}{(N+1) !} \int \tau^{N+1} \tilde{f}(\tau) \phi^{(N+1)}\left(\xi_{\lambda}(\tau)\right) d \tau .
\end{gathered}
$$

Now notice that

$$
\int \tau^{n} \tilde{f}(\tau) d \tau=2 \pi i^{n} f^{(n)}(0) .
$$

Then LHS of (3.22) becomes equal to

$$
\int \frac{1}{\lambda^{2}} e^{i x t / \lambda^{2}} f(x) \phi(t) d x d t-2 \pi \sum_{n=0}^{N} \frac{\left(i \lambda^{2}\right)^{n}}{n !} f^{(n)}(0) \phi^{(n)}(0) .
$$

It is clear that RHS of (3.22) is $o\left(\lambda^{2 N}\right)$.

Remark 3.2. Let us denote non smeared creation and annihilation collective operators as

$$
a_{\lambda}^{ \pm}(k, t):=\frac{1}{\lambda} a^{ \pm}\left(k, t / \lambda^{2}\right)
$$

so that

$$
A_{\lambda}^{+}(t)=\int g(k) a_{\lambda}^{+}(k, t) d k .
$$

The expansion (3.21) for $f_{\lambda}(x, t)$, which can be symbolically rewritten in the form

$$
\frac{1}{\lambda^{2}} e^{i t x / \lambda^{2}}=2 \pi \exp \left(i \lambda^{2} \frac{\partial^{2}}{\partial x \partial t}\right) \delta(x) \delta(t)
$$

induces the corresponding expansion for the non smeared collective operators

$$
a_{\lambda}(k, t)=\sum_{n=0}^{N} \lambda^{n} c_{n}(k, t)+o\left(\lambda^{N}\right)
$$

such that $c_{n}(k, t), n=0,1, \ldots$ satisfy the commutation relations

$$
\left[c_{n}(k, t), c_{m}^{+}(p, \tau)\right]=\delta_{n, m} \frac{2 \pi i^{n}}{n !} \delta^{(n)}(\omega(k)) \delta^{(n)}(\tau-t) \delta(k-p) .
$$

Remark 3.3. It would be interesting to study in that sense one can speak about a spectral decomposition of initial Fock space $\mathcal{F}$ into a family of spaces corresponding to the multipole noise creation and annihilation operators. 
We will use also the notations

$$
c_{0}(k, t) \equiv b(k, t), \quad c_{1}(k, t) \equiv c(k, t), \quad c_{2}(k, t) \equiv d(k, t) .
$$

Here $b(k, t), b^{+}(k, t)$ are the white noise operators:

$$
\left[b(k, t), b^{+}(p, \tau)\right]=2 \pi \delta(\omega(k)) \delta(\tau-t) \delta(k-p)
$$

and $c(k, t), c^{+}(k, t)$ are the dipole noise operators:

$$
\left[c(k, t), c^{+}(p, \tau)\right]=2 \pi i \delta^{\prime}(\omega(k)) \delta^{\prime}(\tau-t) \delta(k-p) .
$$

The second order terms in the expansion (3.23) (we denote their as $d(k, t)$ and $\left.d^{+}(k, t)\right)$ satisfy the commutation relations

$$
\left[d(k, t), d^{+}(p, \tau)\right]=-\pi \delta^{\prime \prime}(\omega(k)) \delta^{\prime \prime}(\tau-t) \delta(k-p) .
$$

For the smeared time dependent operators one has an expansion

$$
A\left(t / \lambda^{2}\right)=\sum_{n=0}^{N} \lambda^{n+1} c_{n}(t)+o\left(\lambda^{N+1}\right) .
$$

The operators $c_{n}(t)$ satisfy the commutation relations

$$
\left[c_{n}(t), c_{m}^{+}(\tau)\right]=\delta_{n, m} \tilde{\gamma}_{n} \delta^{(n)}(\tau-t)
$$

with complex numbers

$$
\tilde{\gamma}_{n}=\frac{(-1)^{n}}{n !} \int_{-\infty}^{\infty} \sigma^{n} d \sigma \int e^{i \sigma \omega(k)}|g(k)|^{2} d k .
$$

The expansion (3.24) holds for any $N \in \mathbb{N}$ in the case then the set of stationary points of function $\omega(k)$ has zero intersection with supp $g(k)$, i.e in the case $|g(k)|^{2} \in S(\Omega)$ for any $\Omega \in \mathbb{R}^{d}$ such that stationary points of $\omega(k)$ do not belong to $\Omega$. The set of stationary points of $\omega(k)$ is defined as $\mathcal{K}:=\left\{k_{0} \mid \operatorname{grad} \omega\left(k_{0}\right)=0\right\}$. The expansion (3.24) holds for $N=1$ and $|g(k)|^{2} \in S\left(\mathbb{R}^{d}\right)$ for $d \geq 3$.

\section{Dipole Noise on the Simplex}

Some modification of Theorem 3.1 is useful for getting the differential equation for the first order correction. This modification is about distributions on the standard simplex (see [2]).

Let us remind the construction for distributions on the standard simplex. Define

$$
\begin{gathered}
C_{0}:=\left\{\phi: \mathbb{R}_{+} \rightarrow \mathbb{C} \mid \phi=0 \text { a.e. }\right\} \\
\tilde{C}_{1}:=\left\{\phi: \mathbb{R}_{+} \rightarrow \mathbb{C} \mid \phi \text { is bounded and left }- \text { continuous at any } t>0\right\} \\
\tilde{C}:=\text { linear span of }\left\{C_{0} \cup \tilde{C}_{1}\right\} .
\end{gathered}
$$


For any $a>0$ define $\delta_{+}(\cdot-a)$ as the unique linear extension of the map

$$
\begin{gathered}
\delta_{+}(\cdot-a): \phi \in \tilde{C}_{1} \rightarrow \phi(a) \\
\delta_{+}(\cdot-a): \phi \in C_{0} \rightarrow 0 .
\end{gathered}
$$

One has the following result: in the sense of distributions on the simplex there exists the limit (see 2] for more discussions)

$$
\lim _{\lambda \rightarrow 0} \frac{1}{\lambda^{2}} e^{i x(t-a) / \lambda^{2}}=\frac{1}{i(x-i 0)} \delta_{+}(t-a) .
$$

In order to introduce the derivative $\delta_{+}^{\prime}(\cdot-a)$ we will define $C_{1}$ as

$$
C_{1}:=\left\{\phi: \mathbb{R}_{+} \rightarrow \mathbb{C} \mid \phi(t)=\sum_{i=1}^{n} \phi_{i}(t) \Theta_{\left[0, a_{i}\right]}(t) \text { for some } n \in \mathbb{N}, 0<a_{i} \leq \infty\right. \text { and }
$$

$\phi_{i}(t)$ is bounded, with bounded and continuous first derivative $\left.\phi_{i}^{\prime}(t)\right\}$.

This definition means that for any $\phi \in C_{1}$ and $a>0$ left-derivative

$$
\phi_{L}^{\prime}(a):=\lim _{\varepsilon \rightarrow-0} \frac{\phi(a+\varepsilon)-\phi(a)}{\varepsilon}
$$

exists. Moreover it is left-continuous and bounded.

With such defined $C_{1}$ we define $C$ as linear span of $\left\{C_{0} \cup C_{1}\right\}$. Clearly $C \subset \tilde{C}$. We will denote the restriction of $\delta_{+}(\cdot-a)$ to $C$ again as $\delta_{+}(\cdot-a)$.

For any $a>0$ define $\delta_{+}^{\prime}(\cdot-a)$ as the unique linear extension of the map

$$
\begin{gathered}
\delta_{+}^{\prime}(\cdot-a): \phi \in C_{1} \rightarrow-\phi_{L}^{\prime}(a) \\
\delta_{+}^{\prime}(\cdot-a): \phi \in C_{0} \rightarrow 0 .
\end{gathered}
$$

With such defined $\delta_{+}^{\prime}(\cdot-a)$ the first order correction to (4.25) (proportional to $\left.\lambda^{2}\right)$ is given by the

Theorem 4.2. One has the following limit in the sense of distributions on the standard simplex ( $a>0$ is a positive number)

$$
\lim _{\lambda \rightarrow 0} \frac{1}{\lambda^{2}}\left(\frac{1}{\lambda^{2}} e^{i x(t-a) / \lambda^{2}}-\frac{1}{i(x-i 0)} \delta_{+}(t-a)\right)=\delta_{+}^{\prime}(t-a) \frac{d}{d x} \frac{1}{x-i 0} .
$$

This means that for arbitrary test functions $f(x) \in S(\mathbb{R})$ and $\phi(t) \in C$ one has the limit

$$
\begin{gathered}
\lim _{\lambda \rightarrow 0} \frac{1}{\lambda^{2}}\left[\frac{1}{\lambda^{2}} \int_{-\infty}^{\infty} d x \int_{0}^{a} d t f(x) \phi(t) e^{i x(t-a) / \lambda^{2}}-\left(\delta_{+}(\cdot-a), \phi\right) \int_{-\infty}^{0} d y \tilde{f}(y)\right] \\
=-\left(\delta_{+}^{\prime}(\cdot-a), \phi\right) \int_{-\infty}^{0} d y y \tilde{f}(y) .
\end{gathered}
$$

Here

$$
\tilde{f}(y)=\int e^{i x y} f(x) d x .
$$


Proof. The Eq. (4.26) clearly holds for $\phi \in C_{0}$. Therefore let us consider the case $\phi \in C_{1}$. It is enough to consider $\phi$ of the form $\phi(x)=\phi_{1}(x) \Theta_{\left[0, a_{1}\right]}(x)$ with $\phi_{1}$ bounded, with bounded and continuous first derivative. In this case $\left(\delta_{+}(\cdot-a), \phi\right)=\phi(a)$ and $\left(\delta_{+}^{\prime}(\cdot-a), \phi\right)=-\phi_{L}^{\prime}(a)$. Making change of the variable $y=(t-a) / \lambda^{2}$ in the first integral one gets

$$
\begin{gathered}
L:=\lim _{\lambda \rightarrow 0} \frac{1}{\lambda^{2}}\left[\int_{-\infty}^{\infty} d x \int_{-a / \lambda^{2}}^{0} d y f(x) \phi\left(\lambda^{2} y+a\right) e^{i x y}-\phi(a) \int_{-\infty}^{0} d y \tilde{f}(y)\right]= \\
\lim _{\lambda \rightarrow 0} \frac{1}{\lambda^{2}}\left[\int_{-a / \lambda^{2}}^{0} d y \tilde{f}(y) \phi\left(\lambda^{2} y+a\right)-\phi(a) \int_{-\infty}^{0} d y \tilde{f}(y)\right] .
\end{gathered}
$$

Let us consider the case $a>a_{1}$. In this case $\phi(a)=0$ and function $\phi\left(\lambda^{2} y+a\right)$ is equal to zero outside of the interval $\left[-a / \lambda^{2},\left(a_{1}-a\right) / \lambda^{2}\right]$. Therefore one has

$$
L=\lim _{\lambda \rightarrow 0} \frac{1}{\lambda^{2}} \int_{-a / \lambda^{2}}^{\left(a_{1}-a\right) / \lambda^{2}} d y \tilde{f}(y) \phi_{1}\left(\lambda^{2} y+a\right)=0
$$

because of boundness of $\phi_{1}$ and Lemma 4.1 In this case $\phi_{L}^{\prime}(a)=0$ so RHS of 4.26) also equal to zero and (4.26) holds.

Now let us consider the case $a \leq a_{1}$. In this case $\phi(a)=\phi_{1}(a)$ and one has

$$
\begin{gathered}
L=\lim _{\lambda \rightarrow 0} \frac{1}{\lambda^{2}}\left[\int_{-a / \lambda^{2}}^{0} d y \tilde{f}(y) \phi_{1}\left(\lambda^{2} y+a\right)-\phi_{1}(a) \int_{-\infty}^{0} d y \tilde{f}(y)\right]= \\
\lim _{\lambda \rightarrow 0} \frac{1}{\lambda^{2}}\left[\int_{-\infty}^{0} d y \tilde{f}(y) \phi_{1}\left(\lambda^{2} y+a\right)-\int_{-\infty}^{-a / \lambda^{2}} d y \tilde{f}(y) \phi_{1}\left(\lambda^{2} y+a\right)-\phi_{1}(a) \int_{-\infty}^{0} d y \tilde{f}(y)\right]= \\
\lim _{\lambda \rightarrow 0} \frac{1}{\lambda^{2}}\left[\int_{-\infty}^{0} d y \tilde{f}(y)\left(\phi_{1}\left(\lambda^{2} y+a\right)-\phi_{1}(a)\right)-\int_{-\infty}^{-a / \lambda^{2}} d y \tilde{f}(y) \phi_{1}\left(\lambda^{2} y+a\right)\right] .
\end{gathered}
$$

Using Lemma 4.1 we get

$$
L=\lim _{\lambda \rightarrow 0} \frac{1}{\lambda^{2}}\left[\int_{-\infty}^{0} d y \tilde{f}(y)\left(\phi_{1}\left(\lambda^{2} y+a\right)-\phi_{1}(a)\right)\right]
$$

Using Lagrange's theorem one gets

$$
\phi_{1}\left(\lambda^{2} y+a\right)-\phi_{1}(a)=\lambda^{2} y \phi_{1}^{\prime}\left(\xi_{\lambda}(y)\right)
$$


with $\xi_{\lambda}(y) \in\left(a+\lambda^{2} y, a\right)$. Therefore

$$
L=\lim _{\lambda \rightarrow 0} \int_{-\infty}^{0} d y y \tilde{f}(y) \phi_{1}^{\prime}\left(\xi_{\lambda}(y)\right) .
$$

Now since $\phi_{1}^{\prime}\left(\xi_{\lambda}(y)\right)$ is bounded and $\tilde{f}(y) \in S(\mathbb{R})$ one can apply Lebesgue's dominated convergence theorem. Finally one has

$$
L=\phi_{1}^{\prime}(a) \int_{-\infty}^{0} y \tilde{f}(y) d y=\phi_{L}^{\prime}(a) \int_{-\infty}^{0} y \tilde{f}(y) d y
$$

Lemma 4.1. For arbitrary test function $f(x) \in S(\mathbb{R})$ and two positive numbers $b>a>0$ one has

$$
\int_{a / \lambda^{2}}^{b / \lambda^{2}} d x f(x)=o\left(\lambda^{2}\right)
$$

Proof. In fact for large enough $x$ and $\forall n \in \mathbb{N} \exists C=$ Const such that $|f(x)| \leq \frac{C}{x^{n}}$. Let us choose $n=3$. Then one has

$$
\lim _{\lambda \rightarrow 0} \frac{1}{\lambda^{2}}\left|\int_{a / \lambda^{2}}^{b / \lambda^{2}} d x f(x)\right| \leq \lim _{\lambda \rightarrow 0} \frac{1}{\lambda^{2}} \int_{a / \lambda^{2}}^{b / \lambda^{2}} d x|f(x)| \leq \lim _{\lambda \rightarrow 0} \frac{C}{\lambda^{2}} \int_{a / \lambda^{2}}^{b / \lambda^{2}} \frac{d x}{x^{3}}=0 .
$$

Remark 4.4. We get the following relation

$$
\frac{1}{\lambda^{2}} e^{i x(t-a) / \lambda^{2}}=\delta_{+}(t-a) \frac{1}{i(x-i 0)}+i \lambda^{2} \delta_{+}^{\prime}(t-a) \frac{d}{d x} \frac{1}{i(x-i 0)}+o\left(\lambda^{2}\right) .
$$

The corresponding expansion for the collective operators has the form

$$
A\left(t / \lambda^{2}\right)=\lambda b(t)+\lambda^{2} c(t)+o\left(\lambda^{2}\right)
$$

with causal commutation relations

$$
\begin{aligned}
& {\left[b(t), b^{+}(\tau)\right]=\gamma_{0} \delta_{+}(\tau-t)} \\
& {\left[c(t), c^{+}(\tau)\right]=\gamma_{1} \delta_{+}^{\prime}(\tau-t) .}
\end{aligned}
$$

Here complex numbers $\gamma_{n}$ are given by

$$
\gamma_{n}=\frac{(-1)^{n}}{n !} \int_{-\infty}^{0} \sigma^{n} d \sigma \int e^{i \sigma \omega(k)}|g(k)|^{2} d k
$$




\section{Corrections to the Green's Functions}

The master field in the standard formulation of the stochastic limit is defined as the limit as $\lambda \rightarrow 0$ of the Wightman correlation functions:

$$
<0\left|A_{\lambda}^{1}\left(t_{1}\right) \ldots A_{\lambda}^{n}\left(t_{n}\right)\right| 0>.
$$

For some models this limit is trivial (equals to zero). However this does not mean that the stochastic limit of such models is trivial because we can consider the limit at $\lambda \rightarrow 0$ of the chronologically ordered correlation functions (Green's functions)

$$
<0\left|T\left(A_{\lambda}^{1}\left(t_{1}\right) \ldots A_{\lambda}^{n}\left(t_{n}\right)\right)\right| 0>.
$$

Here the $T$-product is defined as

$$
T\left(A_{\lambda}^{1}\left(t_{1}\right) \ldots A_{\lambda}^{n}\left(t_{n}\right)\right)=A_{\lambda}^{i_{1}}\left(t_{i_{1}}\right) \ldots A_{\lambda}^{i_{n}}\left(t_{i_{n}}\right)
$$

if $t_{i_{1}} \geq \ldots \geq t_{i_{n}}$.

For the non smeared collective operators $a_{\lambda}^{ \pm}(k, t)$ from Theorem 3.1 one has the following expression for Wigthman functions

$$
\begin{gathered}
<0\left|a_{\lambda}(p, t) a_{\lambda}^{+}(k, \tau)\right| 0>=\frac{1}{\lambda^{2}} e^{i(\tau-t) \omega(p) / \lambda^{2}} \delta(p-k) \\
=2 \pi \delta(\tau-t) \delta(\omega(p)) \delta(p-k)+\lambda^{2} 2 \pi i \delta^{\prime}(\tau-t) \delta^{\prime}(\omega(p)) \delta(p-k)+o\left(\lambda^{2}\right) .
\end{gathered}
$$

On the analogy with Theorem 3.1 it can be proved that

$$
\frac{1}{\lambda^{2}} \Theta(t) e^{i t x / \lambda^{2}}=i \delta(t) \frac{1}{x+i 0}-\lambda^{2} \delta^{\prime}(t) \frac{d}{d x} \frac{1}{x+i 0}+o\left(\lambda^{2}\right) .
$$

Therefore one has for Green's functions

$$
\begin{gathered}
<0\left|T\left(a_{\lambda}(p, t) a_{\lambda}^{+}(k, \tau)\right)\right| 0>=\frac{1}{\lambda^{2}} \Theta(\tau-t) e^{i(\tau-t) \omega(p) / \lambda^{2}} \delta(p-k) \\
=i \delta(\tau-t) \frac{1}{\omega(p)+i 0} \delta(p-k)+\lambda^{2} \delta^{\prime}(\tau-t) \frac{1}{(\omega(p)+i 0)^{2}} \delta(p-k)+o\left(\lambda^{2}\right) .
\end{gathered}
$$

The first term in this expansion was obtained in [2].

\section{A System Interacting with a Reservoir}

In the last sections we derive a generalized quantum stochastic differential equation for corrections to the stochastic limit of the evolution operator $U(t)$. We will apply the dipole noise operators described above for study of the higher order corrections to the stochastic limit. Then we will bring these equations to the normally ordered form and rewrite it in the form of generalized quantum stochastic differential equations.

The study of the evolution operator $U(t)$ describing a system with interaction is very important in statistical physics and quantum field theory.

One of the methods of investigation of behavior of quantum system interacting with a reservoir is the stochastic limit method (see [2] for details). One considers 
quantum models with small coupling constant $\lambda$ for large time $t$. The idea of the method is in systematic application of special limiting procedure $t \rightarrow \infty$ and $\lambda \rightarrow 0$ such that $\lambda^{2} t=\tau=$ Const. The result of such limiting procedure is being called the $\lambda^{2} t$-limit or stochastic limit. The limiting equation for the evolution operator is a quantum stochastic differential equation.

Using this limiting procedure one can study dynamics of real physical system with small but finite coupling constant for large time $t \sim \tau / \lambda^{2}$ without perturbation theory. For some important models dynamics in the stochastic limit approximation became integrable.

The first rigorous result about interaction of a system with a reservoir where the role of $\lambda^{2} t$-rescalling begun emerge is due to Bogoliubov [13. Friedrichs, in the context of the now well known Friedrichs model [14, was lead to consider the scaling limit

$$
t \rightarrow \infty, \quad \lambda \rightarrow 0, \quad \lambda^{2} t=\tau=\text { Const }
$$

by second order perturbation theory. This rescaling was used to derive the master equation by Van Hove [15, Prigogine [16].

The stochastic limit method leads not only to the master equation but also to the equation for the dynamics of the reservoir, see [2, 17, 18.

We will apply the dipole noise operators described above for study of the higher order corrections to the stochastic limit. We will consider the quantum model of $N$-level system (atom) interacting with a boson field.

Let us describe more concretely this model. Let $\mathcal{F}$ as above be the Boson Fock space over the one-particle Hilbert space $L^{2}\left(\mathbb{R}^{d}\right)$. Let $\mathbb{C}^{N}$ be the Hilbert space of $N$-dimensional complex vectors. Then

$$
\Phi=\mathbb{C}^{N} \otimes \mathcal{F}
$$

is the Hilbert space of the model which we consider.

The total Hamiltonian of the model $H_{\lambda}$ is (it acts in $\Phi$ ):

$$
H_{\lambda}=H_{0}+\lambda V .
$$

Here $H_{0}=\mathbf{1} \otimes H_{0 R}+H_{0 S} \otimes \mathbf{1}$ is the free Hamiltonian with

$$
H_{0 R}=\int \omega(k) a^{+}(k) a(k) d k ; \quad k \in \mathbb{R}^{d}
$$

( $d=3$ in the physical space), $\lambda$ is a coupling constant, and the interaction $V$ is given by

$$
V=i\left(D \otimes a^{+}(g)-D^{+} \otimes a(g)\right) .
$$

Here the operators $a^{+}(g)$ and $a(g)$ describe creation and annihilation of bosons; $D$ and $D^{+}$are $N \times N$ - matrices describing transitions between atom levels. The function $g(k)$ is a formfactor (complex valued test function) describing the interaction of the $N$-level system with the reservoir. This Hamiltonian has being applied in quantum optics, tunnelling processes etc (see [19, 20] for details).

The function $\omega(k)$ is a dispersion law. We suppose that the equation $\omega(k)=\omega_{0}$ determines regular surface in $\mathbb{R}^{d}\left(\omega_{0} \in \mathbb{R}\right.$ will be defined below). For example $\omega(k)=\sqrt{k^{2}+m^{2}}$ for massive bosons ( $m$ is the mass of boson) etc. 
Dynamics of the system with $H_{\lambda}$ is being determined by the evolution operator $U_{\lambda}(t)=e^{i t H_{0}} e^{-i t H_{\lambda}}$. It is the solution of the equation (see [8]):

$$
U_{\lambda}(t)=1-i \lambda \int_{0}^{t} V\left(t^{\prime}\right) U_{\lambda}\left(t^{\prime}\right) d t^{\prime} .
$$

Here $V(t)=e^{i t H_{0}} V e^{-i t H_{0}}$ is the interaction operator in the interaction picture.

We will consider two particular types of the model described above. For the first type $N$ is arbitrary natural number and the matrices $D$ and $D^{+}$are connected with $H_{0 S}$ by the following conditions for some $\omega_{0} \in \mathbb{R}$ (the so called rotating wave approximation condition):

$$
\begin{gathered}
e^{i t H_{0 S}} D e^{-i t H_{0 S}}=D e^{-i t \omega_{0}} \\
e^{i t H_{0 S}} D^{+} e^{-i t H_{0 S}}=D^{+} e^{i t \omega_{0}} .
\end{gathered}
$$

Under these conditions one immediately has

$$
V(t)=i\left(D \otimes A^{+}(t)-D^{+} \otimes A(t)\right)
$$

with the time dependent creation and annihilation operators

$$
A^{+}(t)=\int g(k) e^{i\left(\omega(k)-\omega_{0}\right) t} a^{+}(k) d k, \quad A(t)=\int \bar{g}(k) e^{-i\left(\omega(k)-\omega_{0}\right) t} a(k) d k .
$$

After time rescalling $t \rightarrow t / \lambda^{2}$ these operators according to results of the sections 3 and 4 have an asymptotic expansion

$$
A\left(t / \lambda^{2}\right)=\lambda b(t)+\lambda^{2} c(t)+o\left(\lambda^{2}\right)
$$

with white and dipole noise satisfying the (causal) commutation relations which follow from Remark 4.4. These relations are

$$
\begin{aligned}
& {\left[b(t), b^{+}(\tau)\right]=\gamma_{0} \delta_{+}(\tau-t)} \\
& {\left[c(t), c^{+}(\tau)\right]=\gamma_{1} \delta_{+}^{\prime}(\tau-t)}
\end{aligned}
$$

and other commutators are equal to zero. Here $\gamma_{0}$ and $\gamma_{1}$ are complex numbers

$$
\begin{gathered}
\gamma_{0}=-i \int d k \frac{|g(k)|^{2}}{\omega(k)-\omega_{0}-i 0}=\int_{-\infty}^{0} d \sigma \int d k e^{i \sigma\left(\omega(k)-\omega_{0}\right)}|g(k)|^{2} \\
\gamma_{1}=-\int d k \frac{|g(k)|^{2}}{\left(\omega(k)-\omega_{0}-i 0\right)^{2}}=-\int_{-\infty}^{0} d \sigma \sigma \int d k e^{i \sigma\left(\omega(k)-\omega_{0}\right)}|g(k)|^{2} .
\end{gathered}
$$

Usual commutation relations follow from Theorem 3.1

$$
\left[b(t), b^{+}(\tau)\right]=\tilde{\gamma}_{0} \delta(\tau-t)
$$




$$
\left[c(t), c^{+}(\tau)\right]=\tilde{\gamma}_{1} \delta^{\prime}(\tau-t)
$$

with

$$
\begin{gathered}
\tilde{\gamma}_{0}=\int_{-\infty}^{\infty} d \sigma \int d k e^{i \sigma\left(\omega(k)-\omega_{0}\right)}|g(k)|^{2} \\
\tilde{\gamma}_{1}=-\int_{-\infty}^{\infty} d \sigma \sigma \int d k e^{i \sigma\left(\omega(k)-\omega_{0}\right)}|g(k)|^{2} .
\end{gathered}
$$

The second type of the model is the spin-boson model. This model corresponds to the case $N=2$ and no rotating wave approximation condition is assumed. The spin-boson Hamiltonian in the simple but non-trivial case $\varepsilon=0$ has the form (see [17, 21])

$$
H_{\lambda}=-\frac{1}{2} \Delta \sigma_{x} \otimes 1+1 \otimes H_{0 R}+\lambda \sigma_{z} \otimes\left(a(g)+a^{+}(g)\right) .
$$

Here the free Hamiltonian of the boson field $H_{0 R}$ is given by (6.28), $\sigma_{x}, \sigma_{z}$ are the Pauli's matrices, and $\Delta>0$ is a positive number. This model is widely used in physics and chemistry and for example describes a dynamical model of two-level system coupled to an environment (see [17, 21] for example).

For both these models we study asymptotical behavior of the evolution operator $U_{\lambda}\left(t / \lambda^{2}\right)$ with rescalled time. So we study the expansion

$$
U(\lambda, t):=U_{\lambda}\left(t / \lambda^{2}\right)=U_{0}+\lambda U_{1}(t)+o\left(\lambda^{2}\right) .
$$

It was established [2] that $U_{0}$ satisfies the quantum stochastic differential equation. White noise operators play an important role in this consideration. In the present article we show that higher order correction to $U_{0}$ which is $U_{1}$, satisfies the generalized quantum stochastic differential equation. In the derivation of this equation an important role plays the quantum dipole noise.

\section{Higher Order Corrections to the Stochastic Limit of the Vacuum Expectations}

Let us write some results for vacuum expectation of the evolution operator for the models described in previous section.

In [1] Hamiltonians with polynomial self-interaction have been considered. In our case if the system operators $D$ and $D^{+}$are complex numbers then the expression for the vacuum expectation (ABC-formula) has the form

$$
<U_{\lambda}\left(t / \lambda^{2}\right)>=\exp \left(A t+\lambda^{2} B+\lambda^{2} C\left(t / \lambda^{2}\right)\right) .
$$

For the linear model, when $D$ and $D^{+}$are numbers, one has

$$
\begin{gathered}
A=-\gamma_{0} D^{+} D, \quad B=\gamma_{1} D^{+} D \\
C(t)=D^{+} D \int \frac{|g(k)|^{2}}{\left(\omega(k)-\omega_{0}-i 0\right)^{2}} e^{-i\left(\omega(k)-\omega_{0}\right) t} d k
\end{gathered}
$$


and $C(t) \rightarrow 0$ as $t \rightarrow \infty$. Therefore $\lambda^{2} C\left(t / \lambda^{2}\right)=o\left(\lambda^{2}\right)$ and

$$
<U_{\lambda}\left(t / \lambda^{2}\right)>=e^{-\gamma_{0} t D^{+} D}\left(1+\lambda^{2} \gamma_{1} D^{+} D+o\left(\lambda^{2}\right)\right) .
$$

Now let us consider the case then $D$ and $D^{+}$are arbitrary, not necessarily commutative, system operators. Such models were described in the previous section. The expressions for vacuum expectations of the evolution operator for such models were obtained in 22 by direct calculations. For the model with rotating wave approximation one has

$$
\begin{array}{r}
<U_{\lambda}\left(t / \lambda^{2}\right)>=e^{-\gamma_{0} t D^{+} D}\left[1+\lambda^{2} \gamma_{1} D^{+} D\left(1-\gamma_{0} t D^{+} D\right)\right] \\
-\lambda^{2} \gamma_{1} \sum_{k=1}^{\infty} \frac{\left(-\gamma_{0} t\right)^{k}}{k !} \sum_{p=1}^{k}\left(D^{+} D\right)^{p-1} D^{+2} D^{2}\left(D^{+} D\right)^{k-p}+o\left(\lambda^{2}\right) .
\end{array}
$$

In (7.40) and (7.41) $<\cdot>$ means the averaging only over the boson vacuum so that the result is an operator acting in the system Hilbert space $\mathbb{C}^{N}$. Note that if $D$ and $D^{+}$are numbers then the series in (7.40) can be summarized and we obtain (7.39).

For the spin-boson model one has

$$
\begin{gathered}
<U\left(t / \lambda^{2}\right)>_{v a c}=e^{i A_{1} t}\left[1-\lambda^{2}\left(B_{1}+C_{1} t\right)\right] D D^{+} \\
+e^{i A_{2} t}\left[1-\lambda^{2}\left(B_{2}+C_{2} t\right)\right] D^{+} D+o\left(\lambda^{2}\right)
\end{gathered}
$$

with constants $(l=1,2)$

$$
\begin{gathered}
A_{l}=\int d k \frac{|g(k)|^{2}}{\omega_{l}(k)-i 0}, \quad B_{l}=\int d k \frac{|g(k)|^{2}}{\left(\omega_{l}(k)-i 0\right)^{2}}, \\
C_{l}=i A_{l} B_{l}-i \int d k_{1} d k_{2} \frac{\left|g\left(k_{1}\right)\right|^{2}\left|g\left(k_{2}\right)\right|^{2}}{\left(\omega_{l}\left(k_{1}\right)-i 0\right)\left(\omega\left(k_{1}\right)+\omega\left(k_{2}\right)\right)}\left[\frac{1}{\omega_{l}\left(k_{1}\right)-i 0}+\frac{1}{\omega_{l}\left(k_{2}\right)-i 0}\right] .
\end{gathered}
$$

Here $\omega_{1}(k)=\omega(k)-\Delta, \omega_{2}(k)=\omega(k)+\Delta>0$. The matrices $D$ and $D^{+}$in this case are $2 \times 2$-matrices and will be defined in section 9 .

\section{The Normally Ordered Form of the Equation for the Evolution Op- erator}

Let us write the formal equation for the evolution operator (6.38) with the interaction Hamiltonian (6.31) using the expansion (6.32) of the collective operators. Keeping terms of $O(\lambda)$ one has

$$
\frac{\partial U(\lambda, t)}{\partial t}=\left(D\left(b^{+}(t)+\lambda c^{+}(t)\right)-D^{+}(b(t)+\lambda c(t))\right) U(\lambda, t)+\ldots
$$

Here operators $b(t)$ and $c(t)$ are the white and dipole noise with commutation relations (6.33).

Let us expand the time rescalled evolution operator as the series at the coupling constant

$$
U(\lambda, t)=U_{\lambda}\left(t / \lambda^{2}\right)=U_{0}(t)+\lambda U_{1}(t)+\ldots
$$


Then comparing terms of the same order of $\lambda$ in the LHS and RHS of equality (8.42) one obtains a system of stochastic differential equations

$$
\begin{gathered}
\frac{d}{d t} U_{0}(t)=\left(D b^{+}(t)-D^{+} b(t)\right) U_{0}(t) \\
\frac{d}{d t} U_{1}(t)=\left(D c^{+}(t)-D^{+} c(t)\right) U_{0}(t)+\left(D b^{+}(t)-D^{+} b(t)\right) U_{1}(t) .
\end{gathered}
$$

The normally ordered form of equations of such kind is the form in which creation operators are on the left side of the evolution operator and annihilation operators are on the right one. Such form of the equation is convenient for calculation of matrix elements of the evolution operator on coherent (exponential) vectors. The Eq. (8.43) and Eq. (8.44) are equivalent to the following

$$
\begin{gathered}
\frac{d}{d t} U_{0}(t)=D b^{+}(t) U_{0}(t)-D^{+} U_{0}(t) b(t)-D^{+}\left[b(t), U_{0}(t)\right] \\
\frac{d}{d t} U_{1}(t)=D c^{+}(t) U_{0}(t)-D^{+} U_{0}(t) c(t)+D b^{+}(t) U_{1}(t)-D^{+} U_{1}(t) b(t) \\
-D^{+}\left[c(t), U_{0}(t)\right]-D^{+}\left[b(t), U_{1}(t)\right] .
\end{gathered}
$$

Therefore in order to bring the Eq. 8.43) and Eq. (8.44) to the normally ordered form one needs to compute the commutators $\left[b(t), U_{0}(t)\right],\left[c(t), U_{0}(t)\right]$ and $\left[b(t), U_{1}(t)\right]$.

The first commutator had been computed in [2] and equal to

$$
\left[b(t), U_{0}(t)\right]=\gamma_{0} D U_{0}(t)
$$

The second equal to zero because white noise commutes with the dipole noise and $U_{0}(t)$ "consists" only of the white noise. The last commutator can be computed using causal commutation relations (6.33), time consecutive principle and integral form of the equation 8.44

$$
\left[b(t), U_{1}(t)\right]=D \int_{0}^{t} d t_{1}\left[b(t), b^{+}\left(t_{1}\right)\right] U_{1}\left(t_{1}\right)=\gamma_{0} D U_{1}(t) .
$$

Using these commutators one immediately obtains

$$
\begin{gathered}
\frac{d}{d t} U_{0}(t)=D b^{+}(t) U_{0}(t)-D^{+} U_{0}(t) b(t)-\gamma_{0} D^{+} D U_{0}(t) \\
\frac{d}{d t} U_{1}(t)=D c^{+}(t) U_{0}(t)-D^{+} U_{0}(t) c(t) \\
+D b^{+}(t) U_{1}(t)-D^{+} U_{1}(t) b(t)-\gamma_{0} D^{+} D U_{1}(t)
\end{gathered}
$$

We can rewrite Eq. (8.45) and Eq. (8.46) in the forms (1.8) and (1.9) correspondingly. The equation in the form (8.46) we call the generalized quantum stochastic differential equation. It is an important task to develop a theory of equations of such 
form and then to prove the existence and uniqueness of the solution. An interesting problem is to study the unitarity of the solution.

\section{Spin-Boson Hamiltonian}

Let us consider the spin-boson model with Hamiltonian (6.37). The free evolution of $\sigma_{z}$ is

$$
\sigma_{z}(t)=e^{i t H_{s}} \sigma_{z} e^{-i t H_{s}}=e^{i t \Delta} D+e^{-i t \Delta} D^{+}
$$

with

$$
D=\frac{1}{2}\left(\begin{array}{cc}
1 & 1 \\
-1 & -1
\end{array}\right)
$$

It is easy to verify that $D^{2}=D^{+2}=0$ and $\left(D D^{+}\right)^{2}=D D^{+}$. For this interaction (see [17)

$$
V(t)=D \otimes W^{+}(t)+D^{+} \otimes W(t) .
$$

Here $W(t)=A_{1}^{+}(t)+A_{2}(t)$ with

$$
A_{l}(t)=\int d k \bar{g}(k) e^{-i t \omega_{l}(k)} a(k), \quad l=1,2
$$

and $\omega_{1}(k)=\omega(k)-\Delta, \omega_{2}(k)=\omega(k)+\Delta>0$.

We will use the following expansion for the vacuum expectation value of $U(\lambda, t) \equiv U_{\lambda}\left(t / \lambda^{2}\right):$

$$
<U(\lambda, t)>=<U_{0}(t)>+\lambda^{2}<U_{2}(t)>+o\left(\lambda^{2}\right) .
$$

Theorem 9.3. The first correction $f(t) \equiv<U_{2}(t)>$ satisfies the equation

$$
\frac{d f(t)}{d t}=\left(i A_{1} D D^{+}+i A_{2} D^{+} D\right) f(t)-D D^{+} C_{1} e^{i A_{1} t}-D^{+} D C_{2} e^{i A_{2} t},
$$

with constants $(l=1,2)$

$$
\begin{gathered}
A_{l}=\int d k \frac{|g(k)|^{2}}{\omega_{l}(k)-i 0}, \quad B_{l}=\int d k \frac{|g(k)|^{2}}{\left(\omega_{l}(k)-i 0\right)^{2}}, \quad C_{l}=i A_{l} B_{l}-i Z_{l}, \\
Z_{l}=\int d k_{1} d k_{2} \frac{\left|g\left(k_{1}\right)\right|^{2}\left|g\left(k_{2}\right)\right|^{2}}{\left(\omega_{l}\left(k_{1}\right)-i 0\right)\left(\omega\left(k_{1}\right)+\omega\left(k_{2}\right)\right)}\left[\frac{1}{\omega_{l}\left(k_{1}\right)-i 0}+\frac{1}{\omega_{l}\left(k_{2}\right)-i 0}\right]
\end{gathered}
$$

Proof. One has

$$
\begin{gathered}
\frac{\partial<U(\lambda, t)>}{\partial t}=-\frac{i}{\lambda}<V\left(t / \lambda^{2}\right) U(\lambda, t)> \\
=-i<\left[\left(\frac{1}{\lambda} A_{1}\left(t / \lambda^{2}\right) \otimes D+\frac{1}{\lambda} A_{2}\left(t / \lambda^{2}\right) \otimes D^{+}\right), U(\lambda, t)\right]> \\
=-\frac{1}{\lambda^{2}} \int_{0}^{t} d t^{\prime}\left\{\left[A_{1}\left(t / \lambda^{2}\right), A_{1}^{+}\left(t^{\prime} / \lambda^{2}\right)\right] \otimes D D^{+}<U\left(\lambda, t^{\prime}\right)>\right.
\end{gathered}
$$




$$
\begin{gathered}
\left.+<A_{2}\left(t^{\prime} / \lambda^{2}\right) A_{1}\left(t / \lambda^{2}\right) \otimes D D^{+} U\left(\lambda, t^{\prime}\right)>\right\} \\
-\frac{1}{\lambda^{2}} \int_{0}^{t} d t^{\prime}\left\{\left[A_{2}\left(t / \lambda^{2}\right), A_{2}^{+}\left(t^{\prime} / \lambda^{2}\right)\right] \otimes D^{+} D<U\left(\lambda, t^{\prime}\right)>\right. \\
\left.+<A_{1}\left(t^{\prime} / \lambda^{2}\right) A_{2}\left(t / \lambda^{2}\right) \otimes D^{+} D U\left(\lambda, t^{\prime}\right)>\right\} .
\end{gathered}
$$

Let us consider the second term in the first curly bracket. Using the expression

$$
U(\lambda, t)=1-\frac{i}{\lambda} \int_{0}^{t} V\left(t_{1} / \lambda^{2}\right) d t_{1}+\left(-\frac{i}{\lambda}\right)^{2} \int_{0}^{t} d t_{1} \int_{0}^{t_{1}} d t_{2} V\left(t_{1} / \lambda^{2}\right) V\left(t_{2} / \lambda^{2}\right) U\left(\lambda, t_{2}\right)
$$

one gets

$$
\begin{gathered}
\lim _{\lambda \rightarrow 0}-\frac{1}{\lambda^{4}} \int_{0}^{t} d t_{1}<A_{1}\left(t / \lambda^{2}\right) A_{2}\left(t / \lambda^{2}\right) \otimes D D^{+} U\left(\lambda, t^{\prime}\right)> \\
=\lim _{\lambda \rightarrow 0} \frac{1}{\lambda^{6}} \int_{0}^{t} d t_{1} \int_{0}^{t_{1}} d t_{2} \int_{0}^{t_{2}} d t_{3}<A_{1}\left(t / \lambda^{2}\right) A_{2}\left(t_{1} / \lambda^{2}\right) W^{+}\left(t_{2} / \lambda^{2}\right) W\left(t_{3} / \lambda^{2}\right) \\
\otimes\left(D D^{+}\right)^{2} U\left(\lambda, t_{3}\right)>.
\end{gathered}
$$

We suppose that this limit is equal

$$
\begin{gathered}
\lim _{\lambda \rightarrow 0} \frac{1}{\lambda^{6}} \int_{0}^{t} d t_{1} \int_{0}^{t_{1}} d t_{2} \int_{0}^{t_{2}} d t_{3}<A_{1}\left(t / \lambda^{2}\right) A_{2}\left(t_{1} / \lambda^{2}\right) A_{2}^{+}\left(t_{2} / \lambda^{2}\right) A_{1}^{+}\left(t_{3} / \lambda^{2}\right)> \\
\otimes\left(D D^{+}\right)^{2}<U_{0}\left(t_{3}\right)> \\
=\left(D D^{+}\right)^{2} \lim _{\lambda \rightarrow 0} \lim _{\varepsilon \rightarrow+0} \int d k_{1} \int d k_{2}\left|g\left(k_{1}\right)\right|^{2}\left|g\left(k_{2}\right)\right|^{2} \int_{0}^{t / \lambda^{2}} d t_{1} \int_{0}^{t_{1}} d t_{2} \int_{0}^{t_{2}} d t_{3} e^{-i \omega_{1}^{-}\left(k_{1}\right) t / \lambda^{2}-i \omega_{2}\left(k_{2}\right) t_{1}} \\
\times\left\{e^{i \omega_{1}^{-}\left(k_{1}\right) t_{3}+i \omega_{2}\left(k_{2}\right) t_{2}}+e^{\left.i \omega_{1}^{-}\left(k_{2}\right) t_{3}\right)+i \omega_{2}\left(k_{1}\right) t_{2}}\right\}<U_{0}\left(t_{3}\right)>=i D D^{+} Z_{1}<U_{0}(t)>.
\end{gathered}
$$

Here we denote $\omega_{1}^{-}(k):=\omega_{1}(k)-i \varepsilon$.

Using Theorem 4.2 one gets for the first term in the first curly bracket in 9.48)

$$
\begin{gathered}
-\frac{1}{\lambda^{2}} \int_{0}^{t}\left[A_{1}\left(t / \lambda^{2}\right), A_{1}^{+}\left(t^{\prime} / \lambda^{2}\right)\right] \otimes D D^{+}<U\left(\lambda, t^{\prime}\right)>d t^{\prime} \\
=i A_{1} D D^{+}<U_{0}(t)>+i \lambda^{2} A_{1} D D^{+} f(t)-\lambda^{2} D D^{+} B_{1} \frac{d<U_{0}(t)>}{d t}+o\left(\lambda^{2}\right) .
\end{gathered}
$$


Making similar computation with the second curly bracket in (9.48) one gets

$$
\begin{gathered}
\frac{d<U_{0}(t)>}{d t}+\lambda^{2} \frac{d f(t)}{d t}=\left(i A_{1} D D^{+}+i A_{2} D^{+} D\right)<U_{0}(t)> \\
+i \lambda^{2}\left(A_{1} D D^{+}+A_{2} D^{+} D\right) f(t)-\lambda^{2} D D^{+} C_{1} e^{i A_{1} t}-\lambda^{2} D^{+} D C_{2} e^{i A_{2} t} .
\end{gathered}
$$

Let us compare the terms before equal degrees of $\lambda$ in both sides of the last relation. For zero order term one has

$$
\begin{gathered}
\frac{d<U_{0}(t)>}{d t}=\left(i A_{1} D D^{+}+i A_{2} D^{+} D\right)<U_{0}(t)>\Rightarrow \\
<U_{0}(t)>=e^{i A_{1} t} D D^{+}+e^{i A_{2} t} D^{+} D .
\end{gathered}
$$

Now we substitute this solution for $\left\langle U_{0}(t)\right\rangle$ in the terms before $\lambda^{2}$ and obtain the equation

$$
\frac{d f(t)}{d t}=\left(i A_{1} D D^{+}+i A_{2} D^{+} D\right) f(t)-D D^{+} C_{1} e^{i A_{1} t}-D^{+} D C_{2} e^{i A_{2} t} .
$$

This finishes the proof of the theorem.

It is easy to verify that a solution of equation (9.47) is

$$
f(t)=\left[\tilde{B}_{1}-C_{1} t\right] e^{i A_{1} t} D D^{+}+\left[\tilde{B}_{2}-C_{2} t\right] e^{i A_{2} t} D^{+} D .
$$

Here constants $\tilde{B}_{1}, \tilde{B}_{2}$ are determined by initial conditions. In order to obtain (7.41) one should set $\tilde{B}_{1}=-B_{1}, \tilde{B}_{2}=-B_{2}$.

\section{Conclusions}

In the present paper we have considered the operator valued distribution with commutation relations proportional to the derivative of $\delta$-function, the so-called dipole noise operators. We have constructed a representation of these commutation relations in a Fock space with indefinite metric, i.e. in a pseudo-Hilbert space. An interesting problem is to construct a classical analog of this quantum dipole and, more generally, multipole noise.

The multipole noise is interesting not only as a mathematical object but it also plays an important role for applications in physics. In particular, we apply the dipole noise operators to study some models of quantum optics. For the evolution operator of these models we obtain stochastic equations which we call generalized quantum stochastic differential equations. An important open problem is to develop a theory of these generalized quantum stochastic equations, including proving of existence and uniqueness of the solution. An interesting question is to study an analogue of the unitarity of the solution.

The multipole noise describes higher order corrections to the stochastic limit of quantum theory. This is an approximate non-perturbation method which one can apply to study the dynamics of quantum system interacting with a reservoir in the case when coupling constant or density of particles of reservoir is a small parameter and for large time. Therefore it is an important task to apply this approach to other models of quantum theory. 


\section{Acknowledgment}

This work is partially supported by the INTAS $99-00545$ for I. V. and by the INTAS 01/1-200 for A. P. and also by the RFFI 02-01-01084 and the grant of the leading scientific school 00-15-96073.

\section{References}

1. I. Ya. Aref'eva and I. V. Volovich, On the large time behaviour of quantum systems, Inf. Dim. Anal. Quantum Probab. Relat. Topics 3 (2000) 453-482; http://xxx.lanl.gov/abs/quant-ph/9906022

2. L. Accardi, Y. G. Lu and I. V. Volovich, Quantum Theory and Its Stochastic Limit (Springer-Verlag, 2002).

3. L. Accardi, Y. G. Lu and I. V. Volovich, A White Noise Approach to Stochastic Calculus, in Recent Developments in Infinite-Dimensional Analysis and Quantum Probability, Papers in Honour of Takeyuki Hida's 70-th Birthday (Kluwer Acad. Publ., 2000) pp. 3-25.

4. T. Hida, H. H. Kuo, J. Potthoff and L. Streit, White Noise. An Infinite Dimensional Calculus (Kluwer Acad. Publ., 1993).

5. N. Obata, White Noise Calculus and Fock Space, Lect. Notes in Math. 1577 (Springer, New York, 1994).

6. Z. Y. Huang and S. L. Luo, Quantum white noises and free fields, Inf. Dim. Anal. Quantum Probab. Relat. Topics 1 (1998) 69-82.

7. I. V. Volovich, Quantum Dynamics and Black Noise, Talk at the International Conference on Quantum Information, Meijo University, 1998.

8. N. N. Bogoliubov and D. V. Shirkov, Introduction to the Theory of Quantum Fields (Nauka, 1973).

9. T. Ya. Azizov and I. S. Iokhvidov, Fondations of Theory of Linear Operators in Spaces with Indefinite Metric (Nauka, 1986).

10. N. N. Bogoliubov, A. A. Logunov, A. I. Oksak and I. T. Todorov, General Principles of Quantum Field Theory (Nauka, 1987).

11. R. Hudson and K. R. Parthasarathy, Quantum Ito's formula and stochastic evolutions, Comm. Math. Phys. 93 (1984) 301-323.

12. V. S. Vladimirov, Equations of Mathematical Physics (Academic Press, 1973).

13. N. N. Bogoliubov, Problems of Dynamical Theory in Statistical Physics (Gostehizdat, 1946).

14. K. O. Friedrichs, On the perturbation of continuous spectra, Comm. Pure Appl. Math. 1 (1948) 361-406.

15. L. van Hove, Quantum mechanical perturbations giving rise to a transport equation, Physica 21 (1955) 517-540.

16. I. Prigogine, Non-equilibrium Statistical Mechanics (Pergamon, 1963).

17. L. Accardi, S. V. Kozyrev and I. V. Volovich, Dynamics of dissipative twolevel systems in the stochastic approximation, Phys. Rev. A 56 (1997) 2557-2562; http://xxx.lanl.gov/abs/quant-ph/9706021

18. L. Accardi, A. N. Pechen and I. V. Volovich, Quantum stochastic equation for the low density limit, J. Phys. A: Math. Gen. 35 (2002) 4889-4902; http://xxx.lanl.gov/abs/quant-ph/0108112

19. C. Cohen-Tannoudji, J. Dupont-Roc and G. Grienberg, Atom-Photon Interactions, Basic Processes and Applications (John Wiley \& Sons, 1992).

20. D. F. Walls and G. J. Milburn, Quantum Optics (Springer-Verlag, 1994). 
Quantum Multipole Noise

21. A. J. Legett, S. Chakravarty, A. T. Dorsey, M. P. A. Fisher, A. Garg and W. Zwerger, Dynamics of the dissipative two-state system, Rev. Mod. Phys. 59 (1987) 1-85.

22. A. N. Pechen, An approach to calculation of higher order corrections to the stochastic limit in models of quantum theory, Master thesis, Moscow State University, Faculty of Physics (2001). 